04

\title{
Применение модели сфероидальных рассеивателей для определения размеров частиц водных взвесей методами лазерной поляриметрии и динамического рассеяния света
}

\author{
(C) С.Н. Чириков \\ Национальный исследовательский ядерный университет „МИФИ“, \\ 115409 Москва, Россия \\ e-mail: snchirikov@mephi.ru
}

Поступила в редакцию 04.12.2017 г.

Для водных взвесей $\mathrm{ZnO}, \mathrm{CuO}, \mathrm{TiO}_{2}, \mathrm{BaTiO}_{3}$ с частицами неправильной нерегулярной формы приведены результаты измерений размеров частиц дисперсной среды методами лазерной поляриметрии и динамического рассеяния света. При использовании метода лазерной поляриметрии восстановление распределений частиц по размерам проведено в рамках модели сфероидальных рассеивателей. Эта же модель использовалась для коррекции данных, полученных методом динамического рассеяния света. Полученные данные сравнены с результатами, полученными с помощью электронной микроскопии. Показано, что при значениях параметра размера 2-5 коррекция результатов измерений, полученных методом динамического рассеяния света, позволяет уменьшить погрешность определения среднего размера частиц этим методом не более чем на $11 \%$.

DOI: $10.21883 / \mathrm{OS} .2018 .04 .45758 .280-17$

\section{Введение}

Лазерные методы позволяют дистанционно, с высокой чувствительностью и оперативно определять размеры частиц дисперсных сред [1]. В наиболее часто используемых лазерных методах - динамического и статического рассеяния света, как правило, определение размера частиц осуществляется в предположении, что частицы среды имеют сферическую форму, несмотря на то, что частицы большинства дисперсных сред имеют неправильную форму и у них отсутствует воспроизводимость форм. Точность определения размера частиц сферической формы этими методами весьма высока - не хуже 2-3\% (по данным производителей анализаторов частиц). Тем не менее, разброс значений средних размеров, определенных анализаторами, принцип действия которых различен, для монодисперсных взвесей составляет 5-6\%, а для полидисперсных может достигать 10-15\% [2]. Для частиц неправильной формы такой разброс существенно больше $30-60 \%$ [3]. Точность восстановления вида и ширины распределения зависит от ряда факторов: модальности распределения, разницы значений размеров частиц, соответствующих максимумам мод, соотношения между этими максимумами, математического метода, используемого при восстановлении параметров распределения. Большая точность восстановления ширины и вида (модальности) распределения частиц по размерам характерна для метода статического рассеяния света [1]. Замена реальной формы частиц сферической приводит к ошибкам определения дисперсного состава. Восстановление распределения частиц среды по размерам с учетом их формы принципиально возможно на основе измерений поляризационных характеристик рассеянного дисперсной средой излучения (матрицы рассеяния), а не только лишь интенсивности линейно-поляризованной компоненты. Учет конкретных особенностей формы частиц - сложен, требует значительных вычислительных мощностей и практически трудно реализуем. Усилия исследователей в настоящее время направлены на поиск „репрезентативных“ частиц, которые хорошо имитируют рассеивающие свойства реальных дисперсных сред и форму которых можно охарактеризовать двумятремя параметрами [4-7]. К числу таких репрезентативных форм относятся сфероиды (эллипсоиды вращения). Целью настоящей работы являлось сравнение данных определения размеров частиц водных взвесей $\mathrm{ZnO}, \mathrm{CuO}, \mathrm{TiO}_{2}$ и $\mathrm{BaTiO}_{3}$, полученных методами лазерной поляриметрии (ЛП) и динамического рассеяния света (ДРС) в рамках модели сфероидальных рассеивателей.

\section{1. Техника эксперимента}

При приготовлении взвесей порошки перемешивались в дистиллированной воде, полученная смесь фильтровалась и перед измерениями подвергалась ультразвуковой обработке с целью устранения возможных агрегатов. Отсутствие примесей в используемой дистиллированной воде контролировалось с помощью измерения индикатрисы рассеяния от ячейки с водой. При использовании метода лазерной поляриметрии концентрация частиц взвеси подбиралась таким образом, чтобы, с одной стороны, обеспечить удовлетворительное отношение сигнал/шум, а, с другой стороны, чтобы рассе- 

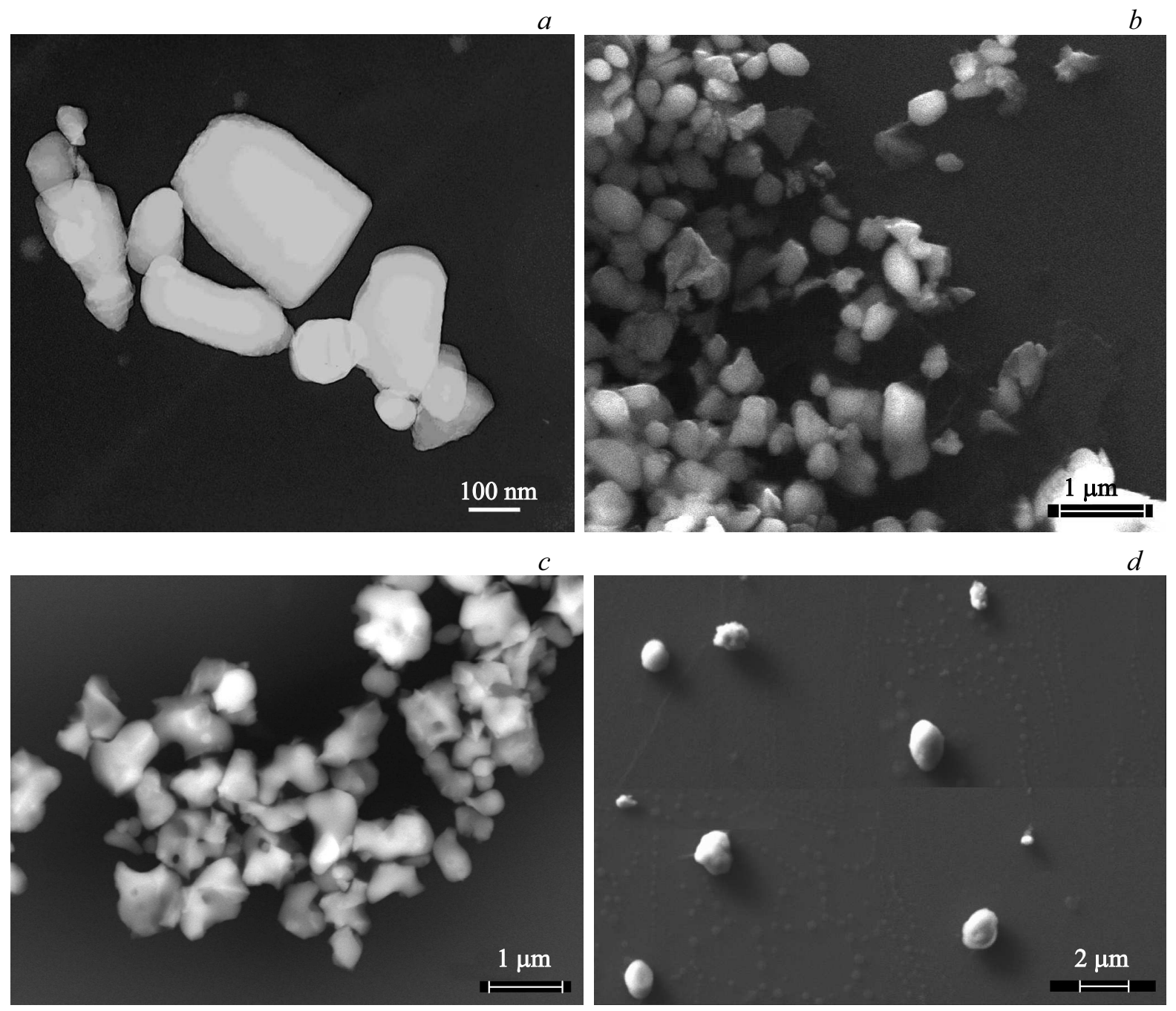

Рис. 1. Микрофотографии частиц (a) $\mathrm{ZnO},(b) \mathrm{CuO},(c) \mathrm{TiO}_{2},(d) \mathrm{BaTiO}_{3}$.

яние можно было считать однократным. Предполагалось, что условия однократности рассеяния выполнены в области, где интенсивность рассеянного излучения линейно возрастает с увеличением концентрации. При определении размеров частиц методом динамического рассеяния света концентрация частиц составляла $(2-6) \cdot 10^{-4} \mathrm{~g} / \mathrm{cm}^{3}$, в этом случае можно не учитывать зависимость коэффициента поступательной диффузии от концентрации.

Микрофотографии частиц $\mathrm{ZnO}, \mathrm{CuO}, \mathrm{TiO}_{2}, \mathrm{BaTiO}_{3}$ исследованных взвесей приведены на рис. 1 . Вид частиц позволяет оценить их сферичность, значения которой составляют 0.7-1. Под сферичностью частицы понимается отношение площади поверхности сферы с объемом, равным объему частицы, к площади поверхности частицы [8].

Определение размеров частиц взвесей методом лазерной поляриметрии основано на измерении зависимостей элементов матрицы рассеяния от угла рассеяния. Матрица рассеяния описывает преобразование состояния поляризации падающего излучения средой. Значения элементов этой матрицы и их зависимости от угла рассеяния определяются распределением по размерам частиц дисперсной среды, их формой и структурой, степенью и характером агломерации, показателем преломления. Для макроскопически изотропной среды, содержащей одинаковое количество хаотически ориентированных рассеивателей и их зеркально-симметричных двойников, матрица рассеяния $F(4 \times 4)$ имеет блочнодиагональный вид [9]. В этом случае элементы $F_{14}$, $F_{41}, F_{24}, F_{42}, F_{31}, F_{32}, F_{13}, F_{23}$ равны нулю, $F_{12}=F_{21}$, $F_{34}=-F_{43}$. Зависимость элемента $F_{11}$ от угла рассеяния $\theta$ описывает индикатрису рассеяния неполяризованного излучения. Для частиц сферической формы $F_{22}=F_{11}$ и $F_{33}=F_{44}$ во всем диапазоне углов рассеяния.

Измерение матриц рассеяния проводилось в диапазоне углов рассеяния $10^{\circ}-155^{\circ}$ с помощью лазерного поляриметра $[10]$, в котором в качестве источника излучения использовался одномодовый $\mathrm{He}-\mathrm{Ne}$ лазер с длиной волны $0.63 \mu \mathrm{m}$ и мощностью $7 \mathrm{~mW}$. 
Для восстановления распределения частиц по размерам экспериментально измеренная матрица рассеяния аппроксимировалась взвешенной суммой рассчитанных теоретически матриц рассеяния модельных (сфероидальных) частиц различного размера и формы (соотношения полуосей):

$$
F_{i j}^{\text {theor }}\left(\theta_{k}\right)=\frac{\sum_{p} \alpha_{p} C_{p}^{\mathrm{sca}} F_{i j}^{p}\left(\theta_{k}\right)}{\sum_{p} \alpha_{p} C_{p}^{\mathrm{sca}}}
$$

где $\theta_{k}-$ угол рассеяния, $\alpha_{p}-$ вклад соответствующего сорта частиц в матрицу рассеяния, $C_{p}^{\text {sca }}-$ сечение рассеяния, а $F_{i j}^{p}-$ матричные элементы $p$ го сорта частиц. Значения весов $\left(\alpha_{p}\right)$, обеспечивающих минимум среднего квадрата отклонений теоретических и экспериментальных данных, и определяли искомое распределение. Число сортов частиц $(p)$ составляло 55 для взвесей $\mathrm{ZnO}$ и $\mathrm{CuO}$, что соответствует разбиению диапазона размеров частиц на 11 смежных областей (в соответствии с гистограммами, полученными по микрофотографиям) и рассмотрению 5 значений соотношения геометрических размеров $\varepsilon=a / b$ (соотношения полуосей сфероидов) в диапазоне значений 0.4-1 (в соответствии с данными, полученными по микрофотографиям) с шагом $\Delta \varepsilon=0.15$. Для взвесей $\mathrm{TiO}_{2}$ и $\mathrm{BaTiO}_{3}$ значения $p$ составили $54(9 \times 6)$ и $48(8 \times 6)$ соответственно при величине $\Delta \varepsilon=0.1$. Для каждого диапазона размеров и значения отношения полуосей рассчитывались элементы матрицы рассеяния и сечения рассеяния. Расчеты проводились с помощью программы, базирующейся на методе Т-матриц, разработанной Мищенко М.И. [9] для ансамбля хаотически ориентированных сфероидов. При расчетах использовалось значение относительного показателя преломления, а частицы дисперсной среды моделировались вытянутыми эллипсоидами вращения. Для определения значений вкладов $\alpha_{i}$, обеспечивающих минимум среднего квадрата отклонений теоретических и экспериментальных данных, использовался алгоритм Левенберга-Марквардта [11].

Для определения размеров частиц использовался также анализатор частиц NanoTrack фирмы Microtrac, принцип действия которого основан на методе динамического рассеяния света. Размеры частиц определяются по ширине функции спектральной плотности напряженности рассеянного назад электрического поля $I(\omega)$. Регистрация рассеянного назад излучения позволяет определять размеры частиц в широком диапазоне значений концентраций, в том числе и при концентрациях, при которых не выполнено условие однократного рассеяния [12]. Применяется гетеродинная схема, где в качестве опорного используется отраженное от поверхности наконечника оптода излучение. При уровне сигнала гетеродина, значительно превышающего уровень рассеянного излучения, спектр мощности фототока воспроизводит спектр $I(\omega)$.
Для невзаимодействующих $N$ одинаковых частиц сферической формы в приближении Релея-Ганса-Дебая спектральная плотность равна [13]:

$$
I(\omega)=N\left(A_{0} V\right)^{2} \frac{D_{T} q^{2}}{\pi\left(\left(\omega-\omega_{0}\right)^{2}+\left(D_{T} q^{2}\right)^{2}\right)},
$$

где $V$ - объем частицы, $A_{0}$ - амплитуда рассеяния на единицу объема рассеивателя, $D_{T}$ - коэффициент поступательной диффузии, $q$ - модуль вектора рассеяния. Модуль вектора рассеяния равен:

$$
q=\frac{4 \pi n_{0}}{\lambda} \sin \left(\frac{\theta}{2}\right)
$$

где $n_{0}-$ показатель преломления жидкости, $\lambda-$ длина волны. Ширина спектра определяется коэффициентом поступательной диффузии:

$$
\Delta \omega_{1 / 2}=D_{T} q^{2}
$$

который для сферических твердых частиц с радиусом $r$, подверженных стоксову трению, равен $[1,13]$ :

$$
D_{T}=\frac{k_{B} T}{6 \pi \eta r}
$$

где $k_{B}-$ константа Больцмана, $T-$ абсолютная температура, $\eta$ - динамическая вязкость среды, в которой движутся частицы. Таким образом, по ширине спектра можно определить размер частиц. Для полидисперсных систем спектральная плотность $I(\omega)$ состоит из нескольких (по числу фракций частиц с различными размерами) лоренцевых кривых (2), наложенных друг на друга, с общим центром при $\omega=\omega_{0}$. Используя математические методы можно по функции спектральной плотности восстановить распределение частиц по размерам. Реализованный в анализаторе NanoTrack математический алгоритм позволяет восстанавливать размеры сферических частиц с точностью $1 \%$ (для частиц полистирола диаметром $100 \mathrm{~nm}$ ). Отметим, что для каждой взвеси методом динамического рассеяния света было проведено 3 измерения.

По совокупности микрофотографий, полученных с помощью электронной микроскопии, для каждой взвеси строились гистограммы распределения частиц по размерам рис. 2 (темные столбцы) и формам. Для этого определялась эффективная площадь частиц и отношение поперечных размеров. При моделировании частиц среды вытянутыми эллипсоидами вращения по этим данным рассчитывался радиус сферы, объем которой равен объему эллипсоида. На рис. 2 под $r$ понимается радиус этой сферы, а под $n_{i} \Delta r_{i}$ - доля частиц с радиусами $r_{i}-r_{i}+\Delta r_{i}$. По гистограммам определялись значения среднего радиуса $r_{m}$ и среднеквадратического отклонения $\sigma$, характеризующего ширину распределения. Полученные значения приведены в табл. 1 (столбцы ЭМ). Данные по распределению частиц по размерам, 

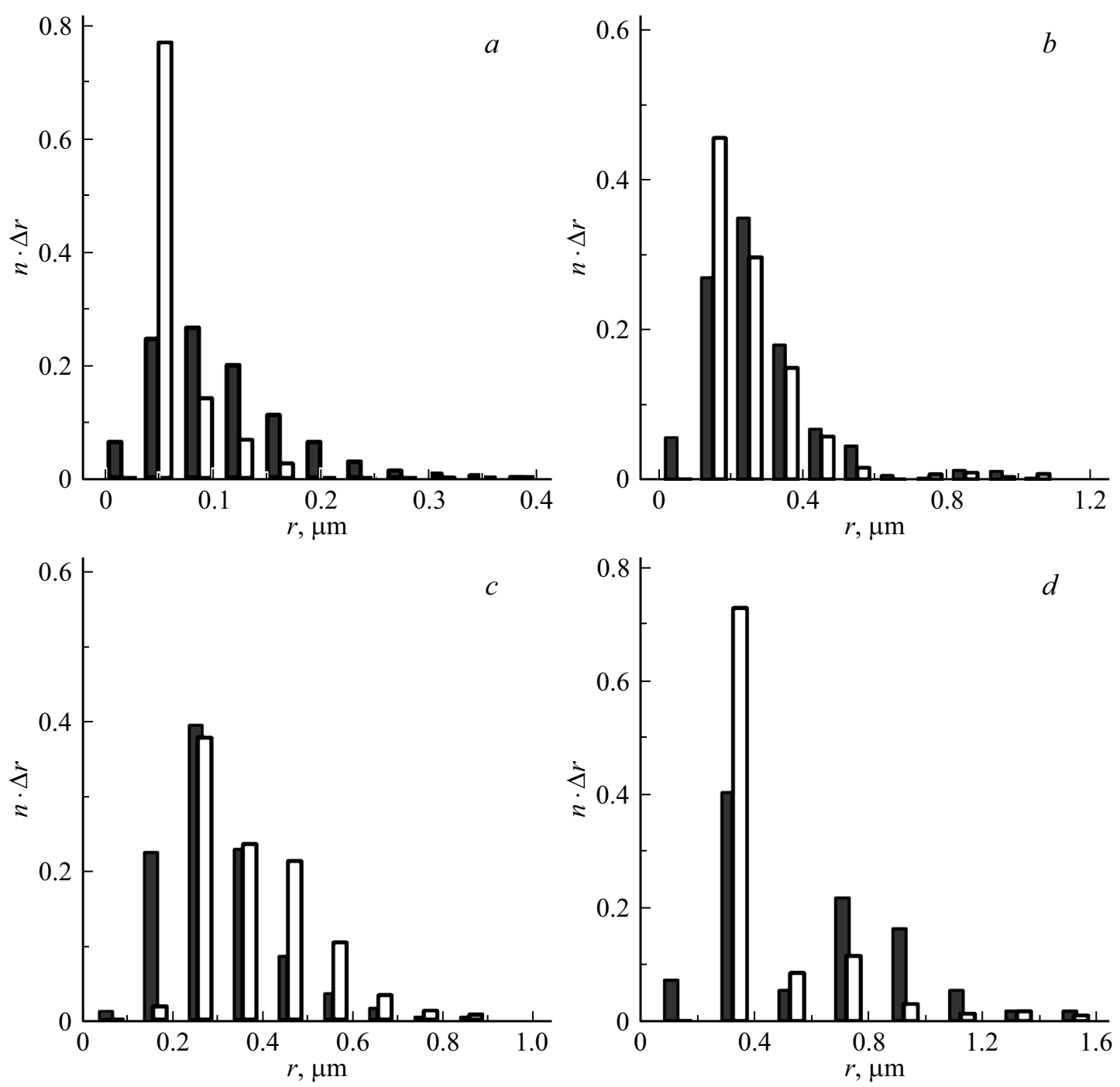

Рис. 2. Гистограммы распределения по размерам частиц (a) $\mathrm{ZnO},(b) \mathrm{CuO},(c) \mathrm{TiO}_{2},(d) \mathrm{BaTiO}_{3}$. Темные столбцы - данные, полученные при обработке микрофотографий. Светлые столбцы - данные, полученные методом лазерной поляриметрии.

полученные методом лазерной поляриметрии и динамического рассеяния света, везде в дальнейшем, если не оговорено, сравниваются с данными, полученными с помощью электронной микроскопии.

\section{2. Экспериментальные результаты}

При восстановлении распределения частиц взвесей по размерам по данным измерений матрицы рассеяния наилучшие результаты были получены при минимизации суммы квадратов отклонений диагональных матричных элементов, а не всех элементов блочно-диагональной матрицы. Восстановленные распределения приведены на рис. 2 (светлые столбцы), а значения среднего размеpa $r_{m}$ и среднеквадратического отклонения $\sigma-$ в табл. 1 (столбцы ЛП). Полученные результаты для всех взвесей кроме $\mathrm{ZnO}$ можно считать удовлетворительными. Значения $r_{m}$ восстанавливаются с погрешностью, не превыша- ющей 30\%, как для одномодального, так и бимодального распределений; величина $\sigma$, характеризующая ширину распределения, в случае одномодального распределения восстанавливается с погрешностью не превышающей $10 \%$, в случае бимодального распределения с погрешностью $27 \%$.

Для взвеси $\mathrm{ZnO}$ в восстановленных распределениях наблюдаются искажения, проявляющиеся в увеличении доли частиц мелкой фракции и уменьшении ширины распределения. Такие искажения будут характерны для восстановленных распределений при значениях параметpa размера $X=2 \pi r / \lambda$ меньших $1-2$. Это объясняется тем, что, во-первых, различия в значениях элемента $F_{11}$ для частиц неправильной формы и смеси сфероидов наиболее просто компенсируются „добавлением“ в смесь сфероидов относительно большого числа частиц мелкой фракции, имеющих малое сечение рассеяния и плавную индикатрису рассеяния (зависимость $F_{11}(\theta)$ ). Заметим, что при минимизации суммы квадратов отклонений 
Таблица 1. Значения параметров распределений

\begin{tabular}{c|c|c|c|c|c|c|c|c|c|c|c|c}
\hline \multirow{2}{*}{ Параметр } & \multicolumn{3}{|c|}{$\mathrm{ZnO}$} & \multicolumn{3}{c|}{$\mathrm{CuO}$} & \multicolumn{3}{c|}{$\mathrm{TiO}_{2}$} & \multicolumn{3}{c}{$\mathrm{BaTiO}_{3}$} \\
\cline { 2 - 13 } & ЭМ & ЛП & ДРС & ЭМ & ЛП & ДРС & ЭМ & ЛП & ДРС & ЭМ & ЛП & ДРС \\
\hline$r_{m}, \mu \mathrm{m}$ & 0.10 & 0.063 & 0.087 & 0.26 & 0.23 & 0.18 & 0.27 & 0.34 & 0.20 & 0.54 & 0.41 & 0.39 \\
$\sigma, \mu \mathrm{m}$ & 0.06 & 0.03 & 0.018 & 0.16 & 0.145 & 0.07 & 0.12 & 0.13 & 0.02 & 0.33 & 0.24 & 0.037 \\
$(a / b)_{m}$ & 0.66 & 0.41 & & 0.71 & 0.49 & & 0.67 & 0.61 & & 0.76 & 0.69 &
\end{tabular}

только лишь элемента $F_{11}$ получаемые при восстановлении значения $r_{m}=0.02 \mu \mathrm{m}$. Во-вторых, зависимости матричных элементов $F_{33}, F_{44}$ от угла рассеяния для сред с частицами неправильной нерегулярной формы аппроксимируются аналогичными зависимостями для смеси сфероидов достаточно хорошо, а зависимости $F_{22}(\theta)$ аппроксимируются хуже. Для частиц неправильной формы значения $F_{22}$ меньше чем для моделирующих их сфероидов с такими же размерами $[9,14,15]$, и эта разница может быть уменьшена при увеличении размеров моделирующих сфероидов. При оптимальных условиях различия зависимостей $F_{22}(\theta)$ для смеси сфероидов и для частиц неправильной формы больше чем различия $F_{33}(\theta), F_{44}(\theta)$ (рис. 3 ). Поэтому основной вклад в величину суммы квадратов отклонений теоретических и экспериментальных значений элементов $F_{22}, F_{33}, F_{44}$ дают отклонения элемента $F_{22}$. Уменьшение размера частиц и увеличение поглощения приводят к стремлению значений $F_{22}$ к единице, уменьшению различий этого элемента для частиц различной формы, и, следовательно, уменьшению величины этого вклада.

Восстановление распределения частиц по размерам по ширине спектра рассеянного излучения является некорректной математической задачей. На ширину получаемых распределений сильно влияет уровень шумов, поэтому в случае слаборассеивающих сред надежно определяется лишь положение максимума распределения. Этим фактом, по-видимому, обусловлены меньшие значения ширин распределений, полученных методом динамического рассеяния света. Возможность восстановления бимодального распределения при соотношении размеров частиц, соответствующих максимумам мод, $1: 2$ для этого метода является предельной (в случае частиц сферической формы) [1], поэтому бимодальность распределения частиц взвеси $\mathrm{BaTiO}_{3}$ не была обнаружена в этих измерениях. Наилучшая точность и воспроизводимость измерений размеров методом динамического рассеяния света для монодисперсных частиц сферической формы достигается при значениях $X \leq 3$ и составляет 5-7\%, а для мономодальных полидисперсных взвесей - 10-12\% [16]. Данные измерений в случае взвеси $\mathrm{ZnO}$, характеризуемой малым значением параметра размера, указывают на сопоставимую погрешность $(13 \%)$, а для других взвесей - на большую погрешность (25-31\%). Возможные причины расхождений могут быть связаны с тем, что восстановление распределений частиц по размерам в методе динамического рассеяния света осуществляется в предположении, что частицы среды имеют сферическую форму. В силу этого, для частиц неправильной формы и сферических частиц могут различаться коэффициенты поступательной и вращательной диффузии, сечения рассеяния, интенсивности обратного рассеяния, что может привести к изменению функции корреляции поля и ширине спектральной плотности напряженности поля рассеянной волны.

\section{3. Коррекция данных измерений методом ДРС}

Проанализируем возможность коррекции данных, полученных методом ДРС, в рамках модели сфероидальных рассеивателей. Для этого сравним корреляционные функции сфер и сфероидов.

Корреляционная функция напряженности поля рассеянной волны $G^{(1)}(\tau)$ и спектральная плотность $I(\omega)$ напряженности связаны теоремой Винера-Хинчина [13]:

$$
I(\omega)=\frac{1}{2 \pi} \int_{-\infty}^{\infty} G^{(1)}(\tau) \exp (i \omega \tau) d \tau,
$$

где $G^{(1)}(\tau)=\left\langle E^{*}(t) E(t+\tau)\right\rangle$, а угловые скобки обозначают усреднение по всему ансамблю частиц.

В предположении, что положения различных рассеивателей некоррелированы, движение центра масс каждой частицы определяется поступательной диффузией и отсутствует корреляция между положением и ориентацией частицы, корреляционная функция напряженности поля рассеянной волны $G^{(1)}(\tau)$ определяется процессами поступательной и вращательной диффузии [13]. Для невзаимодействующих $N$ одинаковых частиц симметричной формы в приближении Релея-Ганса-Дебая функция корреляции $[1,13]$ равна:

$$
\left|G^{(1)}(\tau)\right|=N\left(A_{0} V\right)^{2} \sum_{l=0} B_{i} \exp \left(-\left(D_{T} q^{2}+l(l+1) D_{R}\right) \tau\right),
$$

где суммирование ведется по четным индексам, $D_{R}-$ коэффициент вращательной диффузии, $B_{l}-$ компоненты фактора рассеяния частицы $B_{\text {tot }}=\sum_{l} B_{l}$. Отметим, что величина $\left(A_{0} V\right)^{2} B_{\text {tot }}$ имеет смысл средней интенсивности рассеяния одной частицей.

Основой приближения Релея-Ганса-Дебая является предположение о том, что фазовый сдвиг волны, прошедшей через частицу, незначительно отличается от 

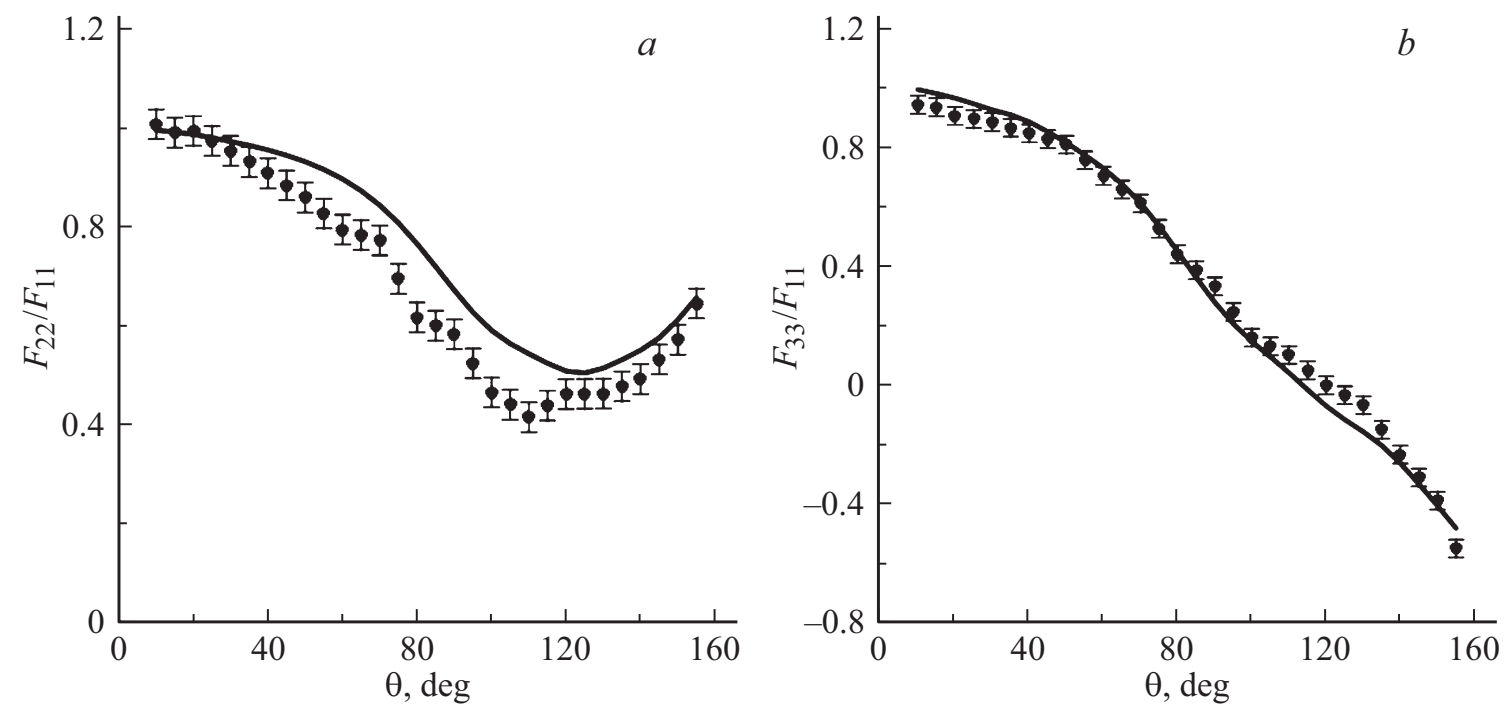

Рис. 3. Зависимости матричных элементов $F_{22}, F_{33}$ от угла рассеяния для взвеси $\mathrm{BaTiO}_{3}$. Точки - экспериментальные данные, линии - расчет для вытянутых эллипсоидов вращения с распределением по размерам, соответствующим минимуму среднеквадратичного отклонения диагональных матричных элементов (рис. $2, d$ ).

фазового сдвига волны, распространяющейся в среде. Авторами [17] было показано, что это приближение может быть использовано и при условии $2 k r\left|n_{r}-1\right| \approx 1$, где $k$ - волновой вектор, $r$ - характерный размер частицы (обычно половина наибольшего размера), $n_{r}-$ относительный показатель преломления. Поэтому это приближение используется в ряде случаев тогда, когда условие $2 k r\left|n_{r}-1\right| \ll 1$ не выполнено.

Средняя интенсивность рассеяния одной частицей в случае вертикальной (по отношению к плоскости рассеяния) поляризации падающей и регистрируемой волны равна:

$$
I_{v v}=\gamma C^{\mathrm{sca}} F_{11}\left(1-2 \frac{F_{12}}{F_{11}}+\frac{F_{22}}{F_{11}}\right),
$$

где $\gamma-$ коэффициент пропорциональности, определяемый расстоянием до фотодетектора.

Перепишем корреляционную функцию напряженности поля для $N$ одинаковых невзаимодействующих частиц в виде:

$$
\left|G^{(1)}(\tau)\right|=N I_{v v} \beta,
$$

где

$$
\beta=\frac{1}{B_{\text {tot }}} \sum_{l=0} B_{l} \exp \left(-\left(D_{T} q^{2}+l(l+1) D_{R}\right) \tau\right) .
$$

Для полидисперсного ансамбля частиц в случае дискретного распределения корреляционная функция поля является суперпозицией функций вида (9):

$$
\left|G^{(1)}(\tau)\right|=\sum_{i} N_{i} I_{v v}^{i} \beta_{i},
$$

где суммирование производится по всем типам частиц, которые могут отличаться как размерами, так и формой.
Тогда модуль нормированной корреляционной функции напряженности поля будет равен $[1,13]$ :

$$
g^{(1)}(\tau)=\frac{G^{(1)}(\tau)}{G^{(1)}(0)}=\sum_{i} w_{i} \beta_{i},
$$

где $w_{i}-$ вклад частиц $i$-го сорта в общую интенсивность рассеяния:

$$
w_{i}=\frac{N_{i} I_{v v}^{i}}{\sum_{i} N_{i} I_{v v}^{i}} .
$$

Коэффициент поступательной диффузии для твердых аксиально-симметричных частиц равен $[1,18]$ :

$$
D_{T}=\frac{k_{B} T}{6 \pi \eta r} H,
$$

где $r$ - характерный размер частицы. Для вытянутых эллипсоидов вращения $r$ равно длине большей полуоси $(b)$, а величина $H$ зависит от отношения полуосей эллипсоида $\varepsilon=a / b[1,19]$ :

$$
H=\left(1-\varepsilon^{2}\right)^{-1 / 2} \ln \left(\frac{1+\sqrt{1-\varepsilon^{2}}}{\varepsilon}\right) .
$$

Коэффициент вращательной диффузии для твердых аксиально-симметричных частиц равен $[1,19]$ :

$$
D_{R}=\frac{3 k_{B} T}{8 \pi \eta r^{3}} P,
$$

где для вытянутых эллипсоидов вращения $[1,19]$ :

$$
P=\frac{\left(2-\varepsilon^{2}\right) H-1}{2\left(1-\varepsilon^{4}\right)} .
$$



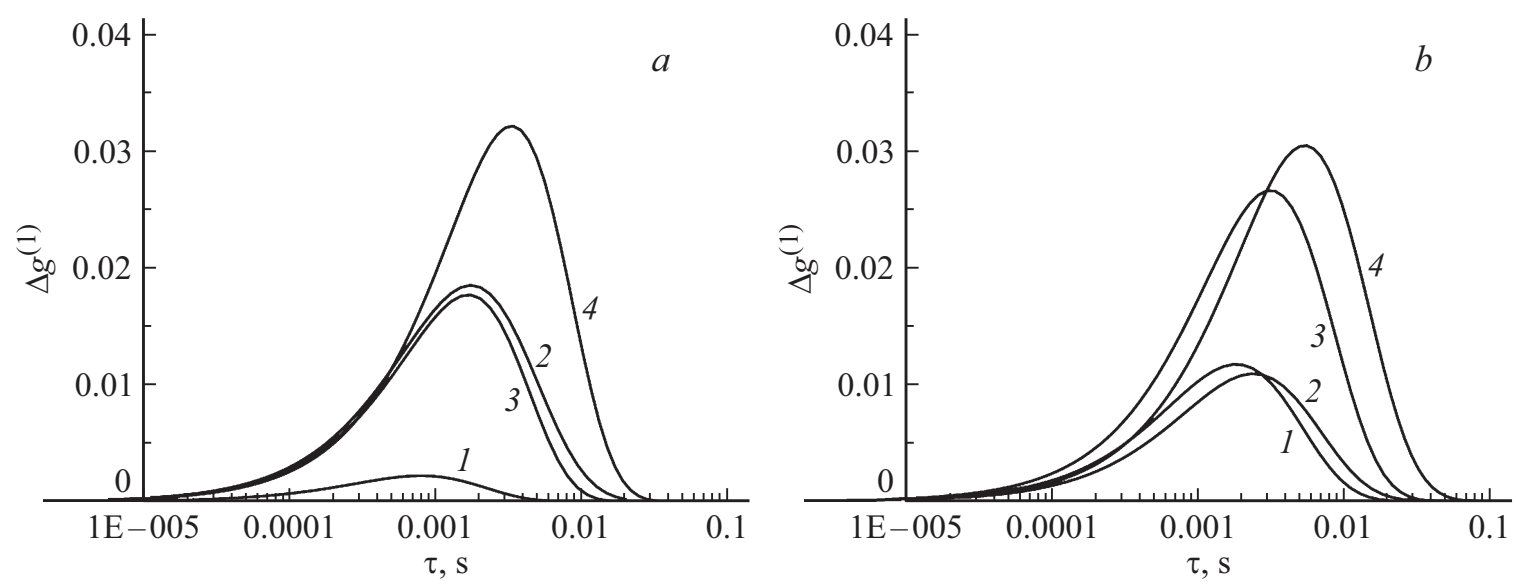

Рис. 4. Разности нормированных корреляционных функций для сфер и сфероидов, рассчитанные для наборов параметров, соответствующих взвесям $\mathrm{ZnO}(1), \mathrm{CuO}(2), \mathrm{TiO}_{2}(3), \mathrm{BaTiO}_{3}(4)$ при ширине распределения, полученной методами ДРС (a) и ЭМ $(b)$.

Сравним нормированные корреляционные функции для полученного методом ДРС распределения частиц для случаев, когда частицы среды имеют сферическую и сфероидальную форму. Значения $\varepsilon$ примем равными средним значениям, определенным по микрофотографиям - 0.66, 0.71, 0.67, 0.76 для взвесей $\mathrm{ZnO}, \mathrm{CuO}$, $\mathrm{TiO}_{2}$ и $\mathrm{BaTiO}_{3}$ соответственно. Результаты расчета сечений рассеяния указывают на пренебрежимо малые различия сечений рассеяния сфер и сфероидов при значениях $X<6$, и незначительные различия элементов $F_{11}$ при значениях $X<3.5$. Отметим, что для случая обратного рассеяния $\left(\theta=180^{\circ}\right)$ значения элемента $F_{12}$ равны нулю, а значения $F_{22}$ для вытянутых эллипсоидов вращения отличаются от единицы не более чем на $5 \%$ для частиц с $X<1.5$. Кроме того, для частиц сферической формы $B_{l}=0$ при $l \neq 0$. Коэффициенты $B_{l}$ для эллипсоидов вращения рассчитывались на основе данных, приведенных в [20]. Отметим, что при значениях $q r \leq 4$ коэффициенты $B_{l}$ при $l \geq 4$ пренебрежимо малы, а при $q r \leq 1.5 B_{2}$ составляет менее $0.7 \%$.

Разность значений нормированных корреляционных функций сфер и сфероидов обусловлена, главным обра-

Таблица 2. Значения среднего размера частиц, полученных методом ДРС

\begin{tabular}{l|c|c|c|c}
\hline \multirow{2}{*}{ Условия определения } & \multicolumn{4}{|c}{$r_{m}, \mu \mathrm{m}$} \\
\cline { 2 - 5 } & $\mathrm{ZnO}$ & $\mathrm{CuO}$ & $\mathrm{TiO}_{2}$ & $\mathrm{BaTiO}_{3}$ \\
\hline $\begin{array}{l}\text { Без коррекции } \\
\text { С коррекцией, }\end{array}$ & 0.087 & 0.18 & 0.20 & 0.39 \\
$\begin{array}{l}\text { при ширине распределения, } \\
\text { полученной методом ДРС } \\
\text { С коррекцией, } \\
\begin{array}{l}\text { при ширине распределения, } \\
\text { полученной методом ЭМ }\end{array}\end{array}$ & 0.087 & 0.19 & 0.21 & 0.42 \\
\end{tabular}

зом, различием значений $\beta$, определяемых коэффициентами поступательной и вращательной диффузии, и приведена на рис. 4 для взвесей $\mathrm{ZnO}, \mathrm{CuO}, \mathrm{TiO}_{2}$ и $\mathrm{BaTiO}_{3}$. Относительное увеличение размера частиц при условии обработки данных в рамках модели сфероидальных (а не сферических) рассеивателей оценивалось из соотношения, полученного дифференцированием функции корреляции $g^{(1)}(\tau)$ для монодисперсных сферических частиц:

$$
\frac{\Delta r}{r}=e \Delta g^{(1)}
$$

При значениях разности $\Delta g^{(1)}=0.002,0.018,0.032$ коррекция данных измерений приводит к относительному увеличению среднего размера частиц на $0.5 \%, 5 \%, 5 \%$, $8.6 \%$ для взвесей $\mathrm{ZnO}, \mathrm{CuO}, \mathrm{TiO}_{2}$ и $\mathrm{BaTiO}_{3}$ соответственно. Наблюдаемые отклонения среднего размера частиц, измеренного методом ДРС, для этих взвесей заметно больше - соответственно $13 \%, 31 \%, 26 \%$ и $28 \%$. Отметим, что при увеличении ширины распределения разность $\Delta g^{(1)}$ будет возрастать. Расчеты показывают, что при ширинах распределения, соответствующих полученным с помощью электронной микроскопии, относительное увеличение размера частиц должно составить $6 \%, 6 \%, 13.6 \%$ и $16 \%$ соответственно. В табл. 2 приведены значения $r_{m}$, полученные после коррекции. Таким образом, коррекция данных измерений среднего размера частиц методом динамического рассеяния света в рамках модели сфероидальных рассеивателей при значениях параметра размера $X<5$, приводит к изменению $r_{m}$ до значений, при которых их отклонения от данных ЭМ составляют $8 \%, 27 \%, 15 \%$ и $17 \%$ для взвесей $\mathrm{ZnO}, \mathrm{CuO}$, $\mathrm{TiO}_{2}$ и $\mathrm{BaTiO}_{3}$ соответственно.

Причиной различия данных, полученных методами электронной микроскопии и ДРС, может быть также асимметрия частиц дисперсной среды. Данные расчета рассеивающих свойств несимметричных частиц, имеющих форму случайных гауссовых сфер и эл- 
липсоидов [5,21], показывают, что для средних значений параметра, характеризующего деформацию сферы или эллипсоида, при малых значениях параметра размера $(\leq 1)$ отсутствует заметное различие сечения рассеяния и интенсивности обратного рассеяния от соответствующих значений для симметричных частиц, а для значений параметра размера $(X=3-5)$, соответствующих окрестности первого максимума зависимости эффективности рассеяния от $X$, для несимметричных частиц характерны меньшие на $10-20 \%$ значения $F_{11}$ и меньшие на $10 \%$ значения эффективности и сечения рассеяния. Для частиц с относительным показателем преломления $n_{r}=1.5(\mathrm{ZnO})$ максимум эффективности рассеяния наблюдается при $X=4$. Для взвеси $\mathrm{ZnO}$ со средним размером частиц, соответствующих значению $X \approx 1$, учет несимметрии формы частиц не может привести к заметной коррекции данных измерений. Для частиц с относительным показателем преломления $n_{r}=2.05$ $\left(\mathrm{TiO}_{2}\right)$ и $n_{r}=2.02+0.34 i(\mathrm{CuO})$ максимум эффективности рассеяния наблюдается при $X=2.5$ и 2.0, соответствующих приблизительно средним значениям размера частиц взвесей $\mathrm{CuO}$ и $\mathrm{TiO}_{2}$. Учет отмеченного выше уменьшения значений $F_{11}$ и сечения рассеяния для взвеси $\mathrm{TiO}_{2}$ приводит к дополнительному изменению размера частиц лишь на $2 \%$.

Шероховатость поверхности частиц тоже может быть причиной различия данных. Моделирование мелкомасштабных шероховатостей поверхности частиц чебышевскими частицами высокого порядка $(n=45)$ и малым значением параметра деформации (0.05), предложенное авторами [22], приводит к изменению сечения рассеяния и интенсивности обратного рассеяния при $n_{r}=1.5(\mathrm{ZnO})$ не более чем на $1 \%$. Для значений параметра размера $X \approx 2-3$ даже в случае частиц $\mathrm{CuO}$ с большим поглощением $\left(n_{r}=2.0+0.34 i\right)$ изменения сечения рассеяния незначительны, а изменения интенсивности обратного рассеяния - не более $4 \%$, что не может привести к заметному сдвигу корреляционной функции.

\section{Заключение}

Использование модели сфероидальных рассеивателей для интерпретации результатов измерений матриц рассеяния дисперсных сред, содержащих неагломерированные частицы неправильной нерегулярной формы со сферичностью больше 0.7 , позволяет с удовлетворительной точностью восстанавливать распределение частиц дисперсной среды по размерам при значениях параметра размера, превышающего 1-2. При меньших значениях параметра размера в восстановленных распределениях возможны искажения, проявляющиеся в увеличении доли частиц мелкой фракции. Метод динамического рассеяния света при малых значениях параметра размера (меньше 1-2) позволяет более точно определять средние размеры частиц взвесей. Применение модели сфероидальных рассеивателей для коррекции данных, полученных методом динамического рассеяния света, позволяет не более чем на $11 \%$ уменьшить погрешность определения среднего размера частиц при значениях параметра размера в диапазоне 2-5.

\section{Список литературы}

[1] $X u$ R. Particle characterization: light scattering methods. N.Y.: Kluwer Academic Publishers, 2002. 398 p.

[2] Delgado A., Matijevic E. // Part. Part. Syst. Character. 1991. V. 8. P. 128-135. doi: 10.1002/ppsc.19910080124

[3] Choi H., Lee W., Kim D., Kumar S., Ha J., Kim S., Lee J. // Korean J. Chem. Eng. 2009. V. 26. N 1. P. 300-305.

[4] Dubovik O., Sinyuk A., Lapyonok T., Holten B.N., Mishchenko M., Yang P., Eck T.F., Volten H., Zande W.J., Leon J.F., Sorokin M., Slutsker I. // J. Geophys. Res.: Atmos. 2006. V. 111. Is. D11. P. D11208-D11238.

[5] Liu J., Yang P., Muinonen K. // J. Quant. Spectrosc. Radiat. Transfer. 2015. V. 161. P. 136-145.

[6] Bi L., Yang P., Kattawar G.W., Kahn R. // Appl. Opt. 2009. V. 48 N 1. P. 114-126.

[7] Bi L., Yang P., Kattawar G.W., Kahn R. // Appl. Opt. 2010. V. 49. N 3. P. 334-343.

[8] Milstein A.B., Richardson J.M. // J. Quant. Spectrosc. Radiat. Transfer. 2015. V. 151. P. 110-122.

[9] Mishchenko M.I. Travis L.D. Lacis A.A. Scattering, Absorption, and Emission of Light by Small Particles. Cambridge: Cambridge University Press, 2002. 448 p.

[10] Ежокин Е.Н., Чириков С.Н. // Опт. и спектр. 2010. Т. 109. № 4. C. 667-674.

[11] Marquardt D. // SIAM J. Appl. Math. 1963. V. 11. Is. 2. P. 431-441.

[12] Wiese H., Horn D. // J. Chem. Phys. 1991. V. 94. Is. 10. P. 6429-6443.

[13] Спектроскопия оптического смешения и корреляция фотонов / Под ред. Камминс Г., Пайк Э.М.: Мир, 1978. 584 с.

[14] Kahnert M., Nousiainen T., Mauno P. // J. Quant. Spectrosc. Radiat. Transfer. 2011. V. 112. P. 1815-1824.

[15] Veihelmann B., Volten H., van der Zande W.J. // Geophys. Res. Lett. 2004. V. 31. Is. 4. P. L04113-L04117.

[16] De Jaeger N., Demeyere H., Finsy R., Sneyers R., Vanderdeelen J., van der Meeren P., van Laethem M. // Part. Part. Syst. Character. 1991. V. 8. P. 179-186.

[17] Barber P.W., Wang D. // Appl. Opt. 1978. V. 17. № 5. P. 797-803.

[18] Berne B.J., Pecora R. Dynamic Light Scattering: with applications to chemistry, biology and physics. N.Y.: Dover Publications Inc., 2000. $372 \mathrm{c}$.

[19] Chu B., Хи R., Dinapoli A. // J. Colloid Interface Sci. 1987. V. 116. № 1. P. 182-195.

[20] Fujime S., Kubota K. // Biophysical Chemistry. 1985. V. 23. Is. $1-2$. P. $1-13$.

[21] Muinonen K., Pieniluoma T. // J. Quant. Spectrosc. Radiat. Transfer. 2011. V. 112. P. 1747-1752.

[22] Rother T., Schmidt K., Wauer J., Shcherbakov V., Gayet J.F. // Appl. Opt. 2006. V. 45. № 23. P. 6030-6037. 